\title{
Human bocavirus induces apoptosis and autophagy in human bronchial epithelial cells
}

\author{
YOU-PING DENG ${ }^{1}$, YING-JUAN LIU ${ }^{2}$, ZHAN-QIU YANG ${ }^{3}$, YAN-JUN WANG ${ }^{1}$, BING-YAN HE ${ }^{1}$ and PIN LIU ${ }^{1}$ \\ ${ }^{1}$ Department of Pediatrics, ${ }^{2}$ Medical Research Center, Zhongnan Hospital, ${ }^{3}$ State Key Laboratory of \\ Virology/Institute of Medical Virology, School of Medicine, Wuhan University, Wuhan, Hubei 430071, P.R. China
}

Received August 24, 2015; Accepted December 23, 2016

DOI: 10.3892/etm.2017.4533

\begin{abstract}
Human bocavirus (HBoV) is classified in the Bocavirus genus within the Parvoviridae family, first identified from children with respiratory diseases. Previous studies have investigated the stimulating effect of $\mathrm{HBoV}$ on cell apoptosis and autophagy. In the present study, human bronchial epithelial cells (HBECs) were utilized to examine the mechanism of $\mathrm{HBoV}$ recombination expressing vector (pWHL-1) on the promotion of cell apoptosis and autophagy. The results from the present study indicated that pWHL-1 inhibited the proliferation of HBECs in a time-dependent manner. Additionally, pWHL-1induced apoptosis, as substantiated by an increased apoptotic rate and presence of autophagosomes. Following pWHL-1 transfection, proliferating cell nuclear antigen, caspase- 3 and B cell lymphoma 2 (Bcl-2) protein expression levels were decreased, with the exception of $\mathrm{Bcl}-2$ associated $\mathrm{x}$ (Bax) protein, which increased. mRNA and protein expression levels of microtubule-associated protein 1A/1B-light chain 3 (LC3) II and autophagy protein 5 were increased in pWHL-1-transfected HBECs, whereas, the mRNA and protein levels of LC3I and sequestosome 1 were decreased. Notably, pWHL-1 also enhanced the activation of p53 and inhibited AKT activation in HBECs. Results from the present study suggest that pWHL-1 induces apoptosis and autophagy, thus providing a novel insight into the effect of $\mathrm{HBoV}$ and its uses in respiratory diseases.
\end{abstract}

\section{Introduction}

Human bocavirus ( $\mathrm{HBoV})$ is classified in the Bocavirus genus within the Parvoviridae family, and was first identified in children with respiratory diseases (1). HBoV infections have been observed worldwide not only in respiratory tract secretions but also in urine, fecal and serum samples $(2,3)$. Newly identified

Correspondence to: Dr You-Ping Deng, Department of Pediatrics, Zhongnan Hospital, Wuhan University, 169 East Lake Road, Wuhan, Hubei 430071, P.R. China

E-mail: dengypwh@126.com

Key words: human bronchial epithelial cells, bocavirus, apoptosis, autophagy, AKT
$\mathrm{HBoV}$ including $\mathrm{HBoV} 2, \mathrm{HBoV} 3$ and $\mathrm{HBoV} 4$ were identified in human stool samples. Previous findings suggest that $\mathrm{HBoV}$ is associated with human diseases and notable pathogenesis; however, there is an inadequate amount of evidence to support this due to the limited establishment of in vitro $\mathrm{HBoV}$ culture systems and animal models (4). HBoV encodes three nonstructural proteins including: NP1, NS1 and NS1-70, in addition to two structural proteins, VP1 and VP2. HBoV NP1 is a nuclear protein, which has an important role in the DNA replication process of $\mathrm{HBoV}$ within the nuclei of infected cells $(5,6)$. It has been reported that NP1 is able to cause cell cycle arrest at $\mathrm{G} 2 / \mathrm{M}$ phase followed by apoptosis, via the mitochondrion pathway in HeLa cells (7). Infection with Bocavirus minute virus of canines (MVC) has also been demonstrated to induce apoptosis, dependent on the replication of the viral genome and arrest G2/M phase in Walter Reed/3873D (WRD) canine cells (8).

The multiple integration of biological and pathological progresses, such as proliferation, differentiation, apoptosis and metabolism, is able to affect the pathogenesis of human diseases. Advanced understanding of the cellular and molecular mechanism of these progresses is important for developing novel diagnostic and therapeutic targets. Previous studies have indicated the cellular bases and roles of autophagy in human health and disease world-wide $(9,10)$. Autophagy is responsible in the in vivo protection against human pathogens that are degraded in vitro by bacteria, viruses and parasites $(11,12)$. Autophagy-related proteins (ATGs), are conserved in mammalian cells and have been indicated to be essential components of the autophagic progress (13). The ubiquitin-like conjugation of ATG5-ATG12 contributes to autophagosome formation and induces LC3 lipidation (LC3I) $(10,14)$. The conversion of cytosolic LC3I to phosphatidylethanolamine-conjugated form (LC3II), a key marker for the autophagosome, indicates the formation of autophagosome (15). SQSTM1, also referred to as p62 protein, is decreased in the presence of autophagy and accumulated in the absent of autophagy, suggesting SQSTM1 may be a marker for autophagy (16).

The molecular mechanism by which $\mathrm{HBoV}$ induces apoptosis and autophagy is not yet well understood. Previous studies have demonstrated that celecoxib and caffeine induced autophagy through inhibition of the PI3K/ATK signaling pathway $(17,18)$. The PI3K/AKT signaling pathway is widely utilized in normal and abnormally activated cells, such as in cancer cells (19). Activation 
of AKT triggers phosphorylation of downstream targets which impacts cell proliferation, apoptosis, migration and autophagy progresses (20). In the present study, human bronchial epithelial cells (HBECs) were used to examine the effect of $\mathrm{HBoV}$ on cell proliferation, apoptosis and autophagy, as well as the mechanism involved. We propose a model of positive regulation of autophagy as part of the host response to $\mathrm{HBoV}$ infection in HBEC.

\section{Materials and methods}

Cell culture. Human bronchial epithelial cells (HBECs; Institute of Biochemistry and Cell Biology, Shanghai, China) at $1 \times 10^{5}$ cells/well were grown on rat-tail collagen I-coated dishes and incubated at $37^{\circ} \mathrm{C}$ in a humidified chamber containing $5 \% \mathrm{CO}_{2}$ for $12 \mathrm{~h}$. Subsequently, HBEC were changed to grow in bronchial epithelial cell basal medium (Clonetics, Co., San Diego, CA, USA) supplemented with $100 \mathrm{mg} / \mathrm{ml}$ penicillin G and $50 \mu \mathrm{g} / \mathrm{ml}$ streptomycin, $0.5 \mu \mathrm{g} / 1$ human epidermal growth factor and $50 \mathrm{mg} / \mathrm{l}$ bovine pituitary extract (all obtained from Invitrogen; Thermo Fisher Scientific, Inc., Waltham, MA, USA) in tissue culture flasks and expanded in the same growth medium. All HBECs were cultured using an incubator and maintained in a humidified atmosphere containing $5 \% \mathrm{CO}_{2}$ at $37^{\circ} \mathrm{C}$.

Lentiviral production and transduction. The $\mathrm{HBoV}$ coding sequence was cloned into a pBluescript SKII vector donated from Yi Li from the Wuhan Engineering Institute (Wuhan, China). The $\mathrm{HBoV}$ recombination expression vector was referred to as pWHL-1. Constructs were subsequently transfected into HEK 293T cells using Lipofectamine 2000 according to the manufacturer's instructions. Viruses were collected following $48 \mathrm{~h}$ transfections and HBECs were infected. HBECs without transfection were used as a control and HBEC with the empty PLKO.1-EGFP vector transfection was used as the negative control (NC).

Cell proliferation assay. HBECs $\left(3 \times 10^{3}\right.$ cells/well) transfected with or without $50 \mathrm{nM}$ pWHL-1 were harvested and plated in 96-well plates. A total of $10 \mu \mathrm{l}$ Cell Counting assay kit-8 (CCK-8) solution (Dojindo Molecular Technologies, Inc., Kumamoto, Japan), according to the manufacturer's protocol, was added to each well and the absorbance was measured at $450 \mathrm{~nm}$ using a microplate reader.

Apoptosis assay. HBECs $\left(3 \times 10^{3}\right.$ cells/well) transfected with or without $50 \mathrm{nM}$ pWHL-1 were harvested and plated in 96-well plates. A cell fixation and permeabilization kit (cat. no. ab185917; Abcam, Cambridge, UK) was used to fix the HBECs in suspension and then permeabilizing the cell membranes, according to the manufacturer's protocol. Subsequently, cells were washed with PBS for 5 min at $25^{\circ} \mathrm{C}$, harvested and stained with $195 \mu 1$ Annexin V-FITC and $5 \mu 1$ propidium iodide (PI; BD Biosciences, San Jose, CA, USA) for $15 \mathrm{~min}$ in the dark at room temperature followed by flow cytometry analysis using BD Accuri ${ }^{\text {TI }}$ C6, version 1.0.264.21 software (BD Biosciences, San Jose, CA, USA).

Immunohistochemistry. HBECs transfected with or without $50 \mathrm{nM}$ pWHL-1 were fixed with $10 \%$ formaldehyde for $48 \mathrm{~h}$ at $25^{\circ} \mathrm{C}$ and blocking of endogenous peroxidases was completed
Table I. Primer sequences used in the present study.

\begin{tabular}{ll}
\hline Gene & \multicolumn{1}{c}{ Primer sequences } \\
\hline LC3I-forward & 5'-TCCGACCGGCCTTTCAAGCAG-3' \\
LC3I-reverse & 5'-GAGAACCTGACCAGAACTCCC \\
& AG-3' \\
LC3II-forward & 5'-GGAAAGCAGCAGTGTACC-3' \\
LC3II-reverse & 5'-CTTTAAGCCGGAAGGCAG-3' \\
ATG5-forward & 5'-GGCTGAGTGAACATCTGAG-3' \\
ATG5-reverse & 5'-CCCAGTTGCCTTATCTGAC-3' \\
SQSTM1-forward & 5'-GGAGTCGGATAACTGTTC-3' \\
SQSTM1-reverse & 5'-GATTCTGGCATCTGTAGG-3' \\
GAPDH-forward & 5'-CACCCACTCCTCCACCTTTG-3' \\
GAPDH-reverse & 5'-CCACCACCCTGTTGCTGTAG-3'
\end{tabular}

LC3, microtubule-associated protein 1A/1B-light chain 3; ATG5, autophagy protein 5; SQSTM1, sequestosome 1.

by soaking slides in a solution of $90 \%$ methanol $/ 3 \% \mathrm{H}_{2} \mathrm{O}_{2}$ for $10 \mathrm{~min}$ at $37^{\circ} \mathrm{C}$. For antigen retrieval, the HBECs were microwaved in $10 \mathrm{mM}$ citrate buffer $(\mathrm{pH} 6.0)$ at $95^{\circ} \mathrm{C}$ for $10 \mathrm{~min}$. HBECs were then incubated with proliferating cell nuclear antigen (PCNA) rabbit monoclonal antibody (cat. no. ab18197; Abcam; 1:1,000) for $1 \mathrm{~h}$ at room temperature after blocking non-specific binding with $10 \%$ normal goat serum (cat. no. 005-000-121; Qcbio Science \& Technologies Co., Ltd., Shanghai, China) in PBS at $37^{\circ} \mathrm{C}$ for $30 \mathrm{~min}$, followed by incubation with goat anti-rabbit biotin-conjugated IgG (cat. no. ab181744; Abcam; $1: 1,000$ ) at $25^{\circ} \mathrm{C}$ for $30 \mathrm{~min}$. HBECs were stained with 3,3'-diaminobenzidine (Shanghai Long Island Biotec., Co., Ltd., Shanghai, China) and hematoxylin staining (Sigma-Aldrich; Merck KGaA). Immunohistochemical signals were calculated with the positive staining of cells using a light microscope (CX41RF; Olympus Corporation, Tokyo, Japan).

Reverse transcription-quantitative polymerase chain reaction $(R T-q P C R)$. Total RNA was isolated from HBECs transfected with or without pWHL-1 using TRIzol Reagent (Invitrogen; Thermo Fisher Scientific Inc.). A total of $1 \mu \mathrm{g}$ RNA was reverse transcribed to synthesize cDNA using Primescript RT Reagent (Takara Biotechnology Co., Ltd., Dalian, China). DNaseI treatment was used to remove genomic DNA. RNA-Primer Mix (12 $\mu \mathrm{l}), 5 x R T$ Reaction Buffer (5 $\mu \mathrm{l}), 25 \mathrm{mM}$ dNTPs $(1 \mu \mathrm{l})$, $25 \mathrm{U} / \mu \mathrm{l}$ R Nase Inhibitor $(1 \mu \mathrm{l}), 200 \mathrm{U} / \mu \mathrm{l}$ M-MLV Rtase $(1 \mu \mathrm{l})$, Oligo $(\mathrm{dt})_{18}(1 \mu \mathrm{l})$ and $\mathrm{ddH}_{2} \mathrm{O}$ (DNase-free; $\left.4 \mu \mathrm{l}\right)$. SYBR-Green qPCR Master Mix (X2; Fermentas; Thermo Fisher Scientific, Inc., Pittsburgh, PA, USA) was used to Real-time PCR performed on an ABI 7500 Real-time PCR system (Applied Biosystems; Thermo Fisher Scientific, Inc., Waltham, MA, USA). SYBRGreen Mix (12.5 $\mu 1)$, forward primer $(0.5 \mu 1)$, reverse primer $(0.5 \mu \mathrm{l}), \mathrm{ddH}_{2} \mathrm{O}(9.5 \mu \mathrm{l})$, cDNA $(2 \mu \mathrm{l})$. The PCR cycling conditions were as follows: $95^{\circ} \mathrm{C}$ for $10 \mathrm{~min}$, followed by 40 cycles at $95^{\circ} \mathrm{C}$ for $15 \mathrm{sec}$ and $60^{\circ} \mathrm{C}$ for $45 \mathrm{sec}$, and a final extension step of $95^{\circ} \mathrm{C}$ for $15 \mathrm{sec}, 60^{\circ} \mathrm{C}$ for $1 \mathrm{~min}, 95^{\circ} \mathrm{C}$ for $15 \mathrm{sec}$ and $60^{\circ} \mathrm{C}$ for $15 \mathrm{sec}$. The primers are listed in Table I. GAPDH mRNA was used as internal control. mRNA expression levels were calculated by the comparative $\Delta \Delta \mathrm{Cq}$ method 

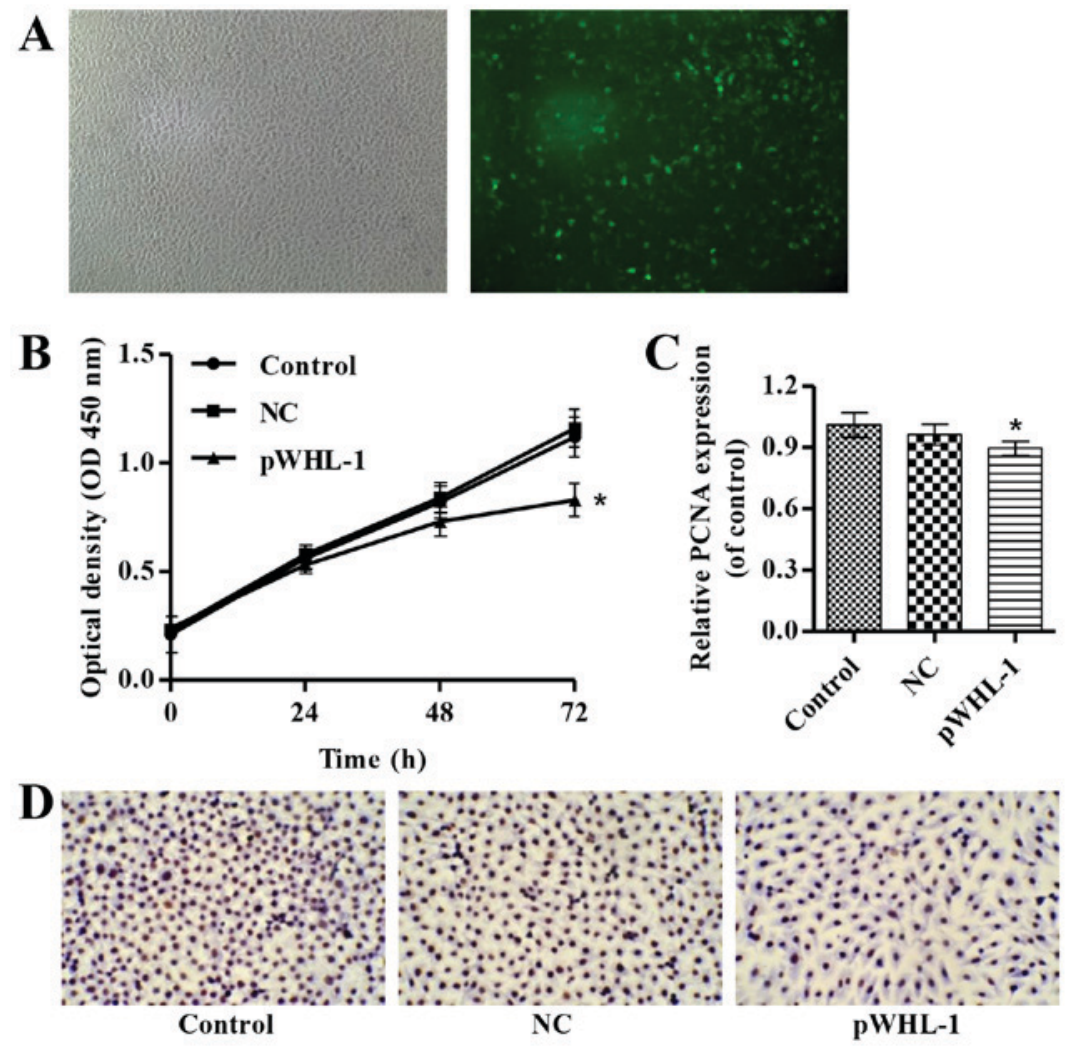

Figure 1. HBoV transfection inhibited HBEC proliferation. HBoV recombination expressing vector was transfected into HBEC for $48 \mathrm{~h}$. (A) Transfection efficiency was evaluated by fluorescence microscopy (left: white light, right: green fluorescence). (B) Proliferation ability was measured using Cell Counting kit-8 analysis. (C) Quantification and (D) representative images of PCNA expression analyzed by immunohistochemistry. The HBoV recombination expression vector was referred to as pWHL-1. HBEC without transfection was used as a control and HBEC with the empty PLKO.1-EGFP vector transfection was used as the NC. "P $<0.05$ vs. NC. Data are presented as the mean \pm standard deviation. HBoV, human bocavirus; HBEC, human bronchial epithelial cells; PCNA, proliferating cell nuclear antigen; NC, negative control. Magnification, x200.

and the fold changes were analyzed by $2^{-\Delta \Delta \mathrm{Cq}}(21)$. The experiment was repeated three times.

Western blot analysis. Total proteins were extracted from HBEC transfected with or without pWHL-1 using RIPA buffer containing $50 \mathrm{mM}$ Tris- $\mathrm{HCl}$, (pH 8.0), $150 \mathrm{mM} \mathrm{NaCl}$, $1 \%$ Nonidet P-40, 0.1\% SDS, 2 mM phenylmethylsulfonyl fluoride, phosphatase and protease inhibitor cocktail (CalbioChem; Merck $\mathrm{KGaA}$ ) at $4^{\circ} \mathrm{C}$ for $20 \mathrm{~min}$, followed by centrifugation at $12,000 \mathrm{x} \mathrm{g}$ for $1 \mathrm{~min}$ at $25^{\circ} \mathrm{C}$. A total of $30 \mu \mathrm{l}$ protein was separated using $12 \%$ SDS-PAGE and transferred to polyvinylidene difluoride membranes. Membranes were blocked in fat-free milk overnight at $4^{\circ} \mathrm{C}$ following three washes with Tris-buffered saline with Tween-20 (Amresco, LLC, Solon, $\mathrm{OH}$, USA) for $5 \mathrm{~min}$ at $25^{\circ} \mathrm{C}$ and subsequently incubated with primary antibodies at $4^{\circ} \mathrm{C}$ overnight. Antibodies used in western blot analysis were as follows: Rabbit monoclonal antibodies for LC3 I/II (cat. no. 4108; 1:1,000; CST Biological Reagents Company Limited, Shanghai, China), SQSTM (cat. no. ab109012; 1:10,000), ATG5 (cat. no. ab109490; 1:1,000), caspase-3 (cat. no. ab32042; 1:1,000), Bcl-2 (cat. no. ab32124; 1:1,000), Bax (cat. no. ab320503; 1:500), p-p53 (cat. no. ab1431; 1:1,000), p-AKT (cat. no. ab38449; 1:1,000), AKT (cat. no. ab8805; 1:500; all purchased from Abcam), GAPDH (cat. no.5174; CST Biological Reagents Company Limited; 1:1,500), and mouse monoclonal antibody for p53 (cat. no. ab1101; Abcam; 1:1,000). Membranes were subsequently washed three times with Tris-buffered saline with Tween-20 (Amresco, LLC), and the peroxidase-conjugated goat anti-rabbit/mouse secondary antibody (1:1,000; cat. no. A0208 and A0216; Beyotime Institute of Biotechnology, Haimen, China) was incubated at $37^{\circ} \mathrm{C}$ for $1 \mathrm{~h}$ and washed three times with Tris-buffered saline with Tween-20 (Amresco, LLC). Immunoreactivity was detected with enhanced chemiluminescence (Merck Millipore; Merck KGaA) and signals were quantified by densitometry (Quantity One software version 4.62; Bio-Rad Laboratories, Inc., Hercules, CA, USA). GAPDH mRNA was used as internal control and the experiment was repeated in triplicate.

Statistical analysis. Data are presented as the mean \pm standard deviation. The paired, two-tailed Student's t-test was used to analyze the significance of difference between groups. Each experiment was performed in triplicate. $\mathrm{P}<0.05$ was considered to indicate a statistically significant difference.

\section{Results}

$H B o V$ suppresses HBEC proliferation. To investigate the biological significance of $\mathrm{HBoV}$ in $\mathrm{HBEC}, \mathrm{HBoV}$ stably expressing cell lines of HBEC were established by pWHL-1 transfection. As indicated in Fig. 1A, fluorescence microscopy evaluation demonstrated a transfection efficiency in HBECs following transfection with pWHL-1. Furthermore, pWHL-1 transfection significantly reduced the proliferation of HBEC 

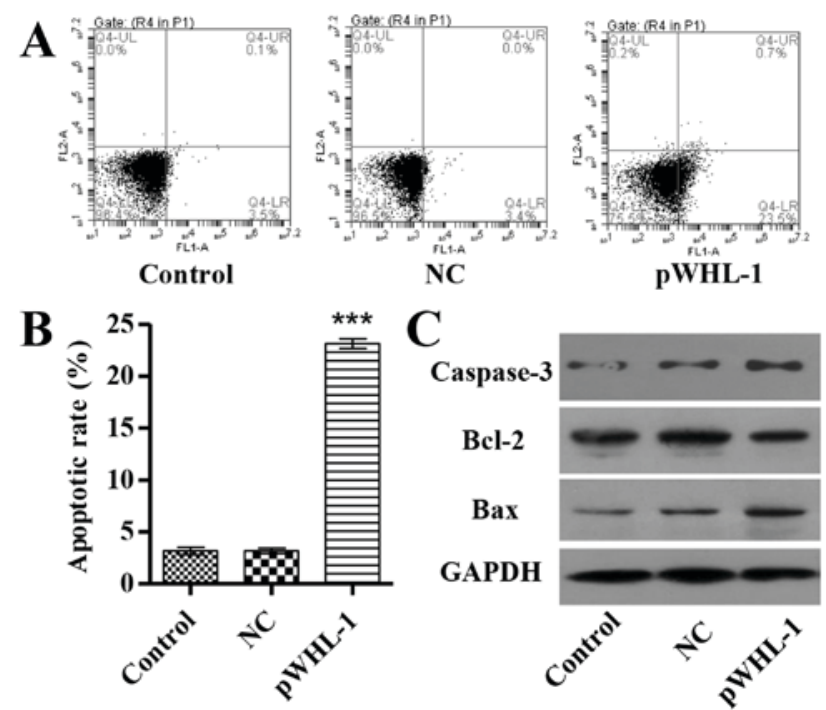

Figure 2. HBoV transfection induced HBEC apoptosis. HBoV recombination expressing vector was transfected into HBEC for $48 \mathrm{~h}$. The HBoV recombination expression vector was referred to as pWHL-1. HBEC without transfection was used as a control and HBEC with the empty PLKO.1-EGFP vector transfection was used as the NC. (A) Representative plot and (B) quantification of apoptotic rate as assessed by flow cytometry. (C) Protein expression levels of caspase-3, Bcl-2 and Bax were detected by western blot analysis. ${ }^{* * * *} \mathrm{P}<0.001$ vs. NC. Data are presented as the mean \pm standard deviation. HBoV, human bocavirus; HBEC, human bronchial epithelial cells; $\mathrm{NC}$, negative control; Bcl-2, B cell lymphoma 2; Bax, Bcl-2 associated protein $\mathrm{X}$

when compared with the control and $\mathrm{NC}$ groups $(\mathrm{P}<0.05$; Fig. 1B). Immunohistochemistry analysis of PCNA, a marker of cell proliferation, indicated that PCNA exhibits significantly decreased levels in pWHL-1 transfected HBEC when compared with the $\mathrm{NC}(\mathrm{P}<0.05$; Fig. $1 \mathrm{C}$ and $\mathrm{D})$.

HBoV promotes HBEC apoptosis. A total of $48 \mathrm{~h}$ after transfection, apoptosis of HBEC was investigated by Annexin V-FITC/PI staining and flow cytometry analysis. The results revealed that pWHL-1 transfection significantly induced HBEC apoptosis by 6.3 -fold when compared with the $\mathrm{NC}$ groups $(\mathrm{P}<0.001$; Fig. 2A and B). To further investigate the mechanism of $\mathrm{HBoV}$ associated with apoptosis of HBEC, three apoptosis associated proteins were also detected in HBEC. Furthermore, the protein expression level of B cell lymphoma (Bcl-2) was significantly decreased $(\mathrm{P}<0.01)$, whereas the protein expression levels of $\mathrm{Bcl}-2$ associated $\mathrm{X}(\mathrm{Bax}, \mathrm{P}<0.01)$ and caspase-3 were significantly increased in HBEC with pWHL-1 transfection, whereas in pWHL-1 transfected HBEC (P<0.01; Fig. 2C).

$H B o V$ induces the autophagy progress in HBEC. To investigate the effect of $\mathrm{HBoV}$ on autophagy of HBEC, a transmission electron microscope was used to observe the cellular morphology of HBEC. The results indicated that pWHL-1 transfected HBEC possessed disordered nuclei, damage of the cellular membrane of organelles, including the mitochondria, and a large number of autophagic vacuoles and autophagosomes in cytoplasm. In comparison with the pWHL-1 transfected HBEC, the control and NC groups showed normally ordered nuclei, abundant and complete cellular membrane of organelles, such as mitochondria, and a fewer number of autophagic vacuoles and autophagosome in cytoplasm (Fig. 3).
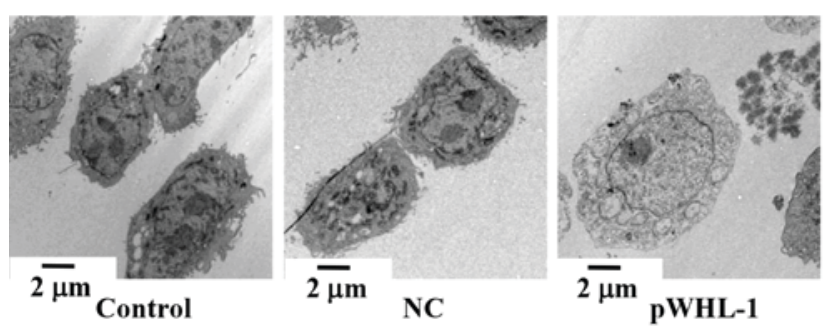

Figure 3. HBoV transfection induced HBEC autophagy. HBoV recombination expressing vector transfected into $\mathrm{HBEC}$ for $48 \mathrm{~h}$. The $\mathrm{HBoV}$ recombination expression vector was referred to as pWHL-1. HBEC without transfection was used as a control and HBEC with the empty PLKO.1-EGFP vector transfection was used as the NC. In comparison to the control and NC groups, pWHL-1 transfected HBEC exhibited disordered nuclei, damage of the cellular membrane in organelles such as mitochondria, and a large number of autophagic vacuoles and autophagosomes were present in the cytoplasm. $\mathrm{HBoV}$, human bocavirus; HBEC, human bronchial epithelial cells; NC, negative control.

Subsequently it considered whether HBoV functionally regulates the autophagy-associated protein in HBEC. To address this question, RT-qPCR and western blot analysis was performed to detect the mRNA and protein expression levels of core proteins involved in the autophagy progress, respectively. These results showed that the mRNA expression levels of LC3I and SQSTM1 were significantly decreased in pWHL-1 transfected HBEC when compared with the control and NC groups, whereas the mRNA expression levels of LC3II and ATG5 were significantly increased in pWHL-1 transfected HBEC when compared with the control and NC groups $(\mathrm{P}<0.01$; Fig. 4A). Similarly, the protein expression levels of LC3I and SQSTM1 were significantly decreased in pWHL-1 transfected HBEC when compared with the control and NC groups, whereas the protein expression levels of LC3II and ATG5 were significantly increased in pWHL-1 transfected HBEC when compared with the control and NC groups $(\mathrm{P}<0.01$; Fig. 4B and $\mathrm{C})$.

$H B o V$ inhibits activation of p53 and AKT. It has been widely recognized that signaling pathways such as p53 and PI3K/AKT pathways are often activated in tumor cells and promote cell proliferation and repress apoptosis and autophagy $(19,20)$. To examine the role of HBoV on p53 and AKT in HBECs, the activation of these proteins was assessed by western blot analysis. In NC HBECs, the P-53/p53 and p-AKT/AKT ratios were unchanged compared with the control cells. In HBECs transfected with pWHL-1, p53 phosphorylation was significantly increased, while AKT phosphorylation was significantly decreased, compared with the NC cells (P<0.01; Fig. 5A and B).

\section{Discussion}

Human bocavirus (HBoV) has been widely regarded to induce apoptosis and autophagy, which is associated with viral pathogenesis (22). In a previous report, human airway epithelia infected with $\mathrm{HBoV}$ were revealed to result in cilia loss and airway epithelial cell hypertrophy; however this was not associated with apoptotic or necrotic cell death (23). Similarly, a previous study demonstrated that $\mathrm{HBoV}$ nonstructural 
A

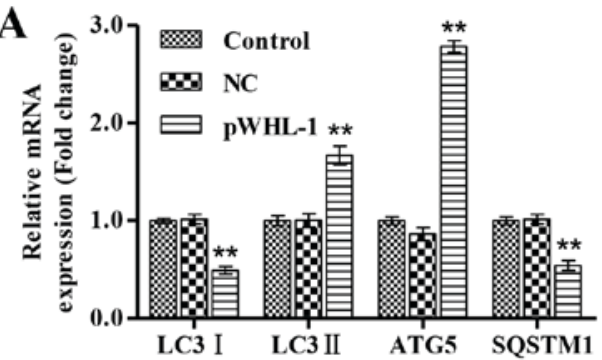

B
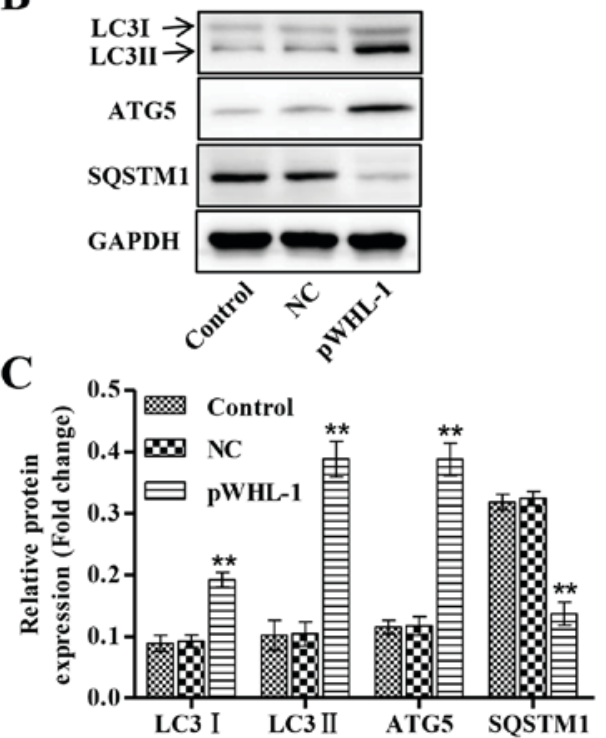

Figure 4. $\mathrm{HBoV}$ infection regulated autophagic core proteins expression. $\mathrm{HBoV}$ recombination expressing vector was transfected into HBEC for $48 \mathrm{~h}$ The HBoV recombination expression vector was referred to as pWHL-1. HBEC without transfection was used as a control and HBEC with the empty PLKO.1-EGFP vector transfection was used as the NC. mRNA and protein expression levels of LC3I, LC3II, ATG5 and SQSTM1 were measured by (A) reverse transcription-quantitative polymerase chain reaction and (B and C) western blot analysis. ${ }^{* *} \mathrm{P}<0.01$ vs. NC. Data are presented as the mean \pm standard deviation. HBoV, human bocavirus; HBEC, human bronchial epithelial cells; NC, negative control; LC3, microtubule-associated protein 1A/1B-light chain 3; ATG5, autophagy protein 5; SQSTM1, sequestosome 1.

protein failed to cause cell death (5). However, in the present study, we demonstrated that $\mathrm{HBoV}$ recombination expressing vector (pWHL-1) induced proliferation inhibition, apoptosis and autophagy in HBECs.

To investigate the effect of $\mathrm{HBoV}$ recombination expressing vector transfection of $\mathrm{HBEC}$, the proliferation of HBEC was measured using aCCK- 8 assay. The results showed that pWHL-1 transfection significantly inhibited the proliferation of HBEC when compared with the control and $\mathrm{NC}$ groups. It has been reported that $\mathrm{HBoV}$ is able to arrest the cell cycle at $\mathrm{S}$ phase; however, cell cycle arrest occurs predominantly at the $\mathrm{G} 2 / \mathrm{M}$ phase, followed by cell apoptosis and proliferation suppression (8). The expression of PCNA was detected in pWHL-1 transfected HBEC by immunohistochemistry assay. The expression of PCNA was significantly decreased in pWHL-1-transfected HBECs when compared with the control and NC groups, which is consisted with the decreased proliferation observed following pWHL-1 transfection in HBECs.

Previous studies have demonstrated that $\mathrm{HBoV}$ induced apoptosis in several cell types, including HeLa, Walter

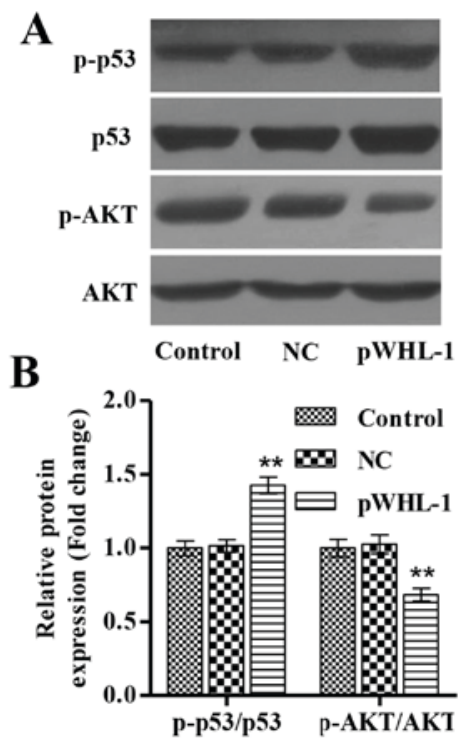

Figure 5. HBoV transfection inhibited the activation of p53 and AKT. HBoV recombination expressing vector was transfected into HBEC for $48 \mathrm{~h}$. The $\mathrm{HBoV}$ recombination expression vector was referred to as pWHL-1. HBEC without transfection was used as a control and HBEC with the empty PLKO.1-EGFP vector transfection was used as the NC. (A and B) Protein expression levels of p-p53, p53, p-AKT and AKT were measured by western blot analysis. ${ }^{* *} \mathrm{P}<0.01$ vs. NC. Data are presented as the mean \pm standard deviation. HBoV, human bocavirus; HBEC, human bronchial epithelial cells; $\mathrm{NC}$, negative control; p53, tumor protein p53; AKT, protein kinase B.

Reed/3873D canine and various types of epithelial cells $(7,8)$. Therefore, the predominant focus of the present study was on the effect of pWHL-1 on HBEC apoptosis. The present findings identified that pWHL-1 significantly induced apoptosis of HBECs and regulated the protein expression of caspase-3, Bax and Bcl-2. Western blot analysis demonstrated that the protein expression levels of caspase-3 and Bax were increased, whereas the protein expression levels of $\mathrm{Bcl}-2$ were decreased in pWHL-1-transfected HBECs compared with control and $\mathrm{NC}$ groups, suggesting that $\mathrm{HBoV}$ may induce apoptosis through a mitochondrion-mediated pathway and increase the ratio of $\mathrm{Bax} / \mathrm{Bcl}-2$. In agreement with our findings, a previous study reported that $\mathrm{HBoV}$ also induced cell apoptosis through the activation of caspase- 3 and caspase- 9 and an increase of $\mathrm{Bax} / \mathrm{Bcl}-2$ ratio was observed (7).

Autophagy is a crucial component of the cellular stress adaptation response that maintains mammalian homeostasis (24). Autophagosome formation proceeds through a series of stages and has various roles in cancer development and progression, and is involved in the proliferation of normal cells (25). In the present study, pWHL-1 infection was indicated to induce autophagy, as evidenced by the presence of disordered nuclei, damage of the cellular membrane of organelles, such as mitochondria, and large numbers of autophagic vacuoles and autophagosome observed in cytoplasm. In addition, specific autophagy associated proteins were also detected in HBEC. RT-qPCR and western blot analysis demonstrated that the mRNA and protein expression of LC3II and ATG5 were significantly increased, whereas LC3I and SQSTM1 mRNA and protein expression levels were significantly decreased in pWHL-1-transfected HBECs. An increased ratio of LC3II/LC3I is a marker of an enhancement in 
autophagosomes formation, whereas SQSTM1 expression is negatively correlated with autophagy (26). ATG5 is involved in the early stage of autophagosome formation and has multiple functions in various physiological contexts (27).

The molecular mechanism by which $\mathrm{HBoV}$ induces autophagy in HBEC is not yet fully understood. Autophagy and apoptosis may coadjust through p53 and PI3K/AKT signaling $(28,29)$. The p53 and PI3K/AKT signaling pathway are two well-known pathways involved in the regulation of autophagy (30). Both pathways are associated with tumorigenesis and activated in a number of cancers (19). Yersinia pestis infection of HBECs has been associated with the negative regulation of autophagy via the observed decrease exhibited of p53 cytoplasmic localization and PI3K/AKT activation (31). Furthermore, plumbagin has been demonstrated to induce autophagy via the inhibition of the PI3K/AKT pathway in human non-small cell lung cancer cells (32) In the present study, the activation of p53 was significantly increased; however, AKT protein activation via phosphorylation was significantly decreased in pWHL-1-transfected HBECs when compared with the control and $\mathrm{NC}$ groups, indicating that $\mathrm{HBoV}$ induced HBEC autophagy predominantly through enhancing p53 activation and blocking AKT activation.

In conclusion, the present study demonstrated that $\mathrm{HBoV}$ promoted the inhibition of proliferation, apoptosis and autophagy in HBEC and the apoptosis and autophagy were associated with the regulation of p53 and AKT. The present study may be useful to address the precise effect of $\mathrm{HBoV}$ in HBEC proliferation, apoptosis and autophagy and to delineate the molecular mechanism of $\mathrm{HBoV}$ in respiratory diseases.

\section{Acknowledgements}

The present study was supported by the National Natural Science Foundation of China (grant no. 81101306).

\section{References}

1. Allander T, Tammi MT, Eriksson M, Bjerkner A, Tiveljung-Lindell A and Andersson B: Cloning of a human parvovirus by molecular screening of respiratory tract samples. Proc Natl Acad Sci USA 102: 12891-12896, 2005.

2. Lindner J and Modrow S: Human bocavirus-a novel parvovirus to infect humans. Intervirology 51: 116-122, 2008.

3. Allander T, Jartti T, Gupta S, Niesters HG, Lehtinen P, Osterback R, Vuorinen $\mathrm{T}$, Waris $\mathrm{M}$, Bjerkner A, Tiveljung-Lindell A, et al: Human bocavirus and acute wheezing in children. Clin Infect Dis 44: 904-910, 2007.

4. Mitui MT, Tabib SM, Matsumoto T, Khanam W, Ahmed S, Mori D, Akhter N, Yamada K, Kabir L, Nishizono A, et al: Detection of human bocavirus in the cerebrospinal fluid of children with encephalitis. Clin Infect Dis 54: 964-967, 2012.

5. Chen AY, Cheng F, Lou S, Luo Y, Liu Z, Delwart E, Pintel D and Qiu J: Characterization of the gene expression profile of human bocavirus. Virology 403: 145-154, 2010.

6. Sun Y, Chen AY, Cheng F, Guan W, Johnson FB and Qiu J: Molecular characterization of infectious clones of the minute virus of canines reveals unique features of bocaviruses. J Virol 83: 3956-3967, 2009.

7. Sun B, Cai Y, Li Y, Li J, Liu K, Li Y and Yang Y: The nonstructural protein NP1 of human bocavirus 1 induces cell cycle arrest and apoptosis in Hela cells. Virology 440: 75-83, 2013.

8. Chen AY, Luo Y, Cheng F, Sun Y and Qiu J: Bocavirus infection induces mitochondrion-mediated apoptosis and cell cycle arrest at G2/M phase. J Virol 84: 5615-5626, 2010.

9. Levine B and Kroemer G: Autophagy in the pathogenesis of disease. Cell 132: 27-42, 2008.
10. Ravikumar B, Sarkar S, Davies JE, Futter M, Garcia-Arencibia M, Green-Thompson ZW, Jimenez-Sanchez M, Korolchuk VI, Lichtenberg M, Luo S, et al: Regulation of mammalian autophagy in physiology and pathophysiology. Physiol Rev 90: 1383-1435, 2010.

11. Rubinsztein DC, Codogno P and Levine B: Autophagy modulation as a potential therapeutic target for diverse diseases. Nat Rev Drug Discov 11: 709-730, 2012.

12. Tallóczy Z, Jiang W, Virgin HW IV, Leib DA, Scheuner D, Kaufman RJ, Eskelinen EL and Levine B: Regulation of starvation- and virus-induced autophagy by the eIF2alpha kinase signaling pathway. Proc Natl Acad Sci USA 99: 190-195, 2002.

13. Burman C and Ktistakis NT: Autophagosome formation in mammalian cells. Semin Immunopathol 32: 397-413, 2010.

14. Ohsumi Y: Molecular dissection of autophagy: Two ubiquitin-like systems. Nat Rev Mol Cell Biol 2: 211-216, 2001.

15. Mizushima N and Komatsu M: Autophagy: Renovation of cells and tissues. Cell 147: 728-741, 2011.

16. Bjørkøy G, Lamark T, Pankiv S, Øvervatn A, Brech A and Johansen T: Monitoring autophagic degradation of p62/SQSTM1. Method Enzymol 452: 181-197, 2009.

17. Liu M, Li CM, Chen ZF, Ji R, Guo QH, Li Q, Zhang HL and Zhou YN: Celecoxib regulates apoptosis and autophagy via the PI3K/Akt signaling pathway in SGC-7901 gastric cancer cells. Int J Mol Med 33: 1451-1458, 2014

18. Saiki S, Sasazawa Y, Imamichi Y, Kawajiri S, Fujimaki T, Tanida I, Kobayashi H, Sato F, Sato S, Ishikawa K, et al: Caffeine induces apoptosis by enhancement of autophagy via PI3K/Akt/mTOR/p70S6K inhibition. Autophagy 7: 176-187,2011.

19. Luo J, Manning BD and Cantley LC: Targeting the PI3K-Akt pathway in human cancer: Rationale and promise. Cancer Cell 4: 257-262, 2003

20. Annovazzi L, Mellai M, Caldera V, Valente G, Tessitore L and Schiffer D: mTOR, S6 and AKT expression in relation to proliferation and apoptosis/autophagy in glioma. Anticancer Res 29: 3087-3094, 2009.

21. Livak KJ and Schmittgen TD: Analysis of relative gene expression data using real-time quantitative PCR and the 2(-Delta Delta C(T)) Method. Methods 25: 402-408, 2001.

22. Chen AY and Qiu J: Parvovirus infection-induced cell death and cell cycle arrest. Future Virol 5: 731-743, 2010.

23. Huang Q, Deng X, Yan Z, Cheng F, Luo Y, Shen W, Lei-Butters DC, Chen AY, Li Y, Tang L, et al: Establishment of a reverse genetics system for studying human bocavirus in human airway epithelia. PLoS Pathog 8: e1002899, 2012.

24. Allan LA and Clarke PR: Apoptosis and autophagy: Regulation of caspase-9 by phosphorylation. FEBS J 276: 6063-6073, 2009.

25. White E: Deconvoluting the context-dependent role for autophagy in cancer. Nat Rev Cancer 12: 401-410, 2012.

26. Wang L, Dong Z, Huang B, Zhao B, Wang H, Zhao J, Kung H, Zhang S and Miao J: Distinct patterns of autophagy evoked by two benzoxazine derivatives in vascular endothelial cells. Autophagy 6: 1115-1124, 2010.

27. Mizushima N, Ohsumi Y and Yoshimori T: Autophagosome formation in mammalian cells. Cell Struct Funct 27: 421-429, 2002.

28. Liu J, Lin Y, Yang H, Deng Q, Chen G and He J: The expression of p33(ING1), p53, and autophagy-related gene Beclin1 in patients with non-small cell lung cancer. Tumor Biol 32: 1113-1121, 2011.

29. Cheng Y, Ren X, Zhang Y, Patel R, Sharma A, Wu H, Robertson GP, Yan L, Rubin E and Yang JM: eEF-2 kinase dictates cross-talk between autophagy and apoptosis induced by Akt Inhibition, thereby modulating cytotoxicity of novel Akt inhibitor MK-2206. Cancer Res 71: 2654-2663, 2011.

30. Yuan L, Wei S, Wang J and Liu X: Isoorientin induces apoptosis and autophagy simultaneously by reactive oxygen species (ROS)-related p53, PI3K/Akt, JNK, and p38 signaling pathways in HepG2 cancer cells. J Agric Food Chem 62: 5390-5400, 2014.

31. Alem F, Yao K, Lane D, Calvert V, Petricoin EF, Kramer L, Hale ML, Bavari S, Panchal RG and Hakami RM: Host response during Yersinia pestis infection of human bronchial epithelial cells involves negative regulation of autophagy and suggests a modulation of survival-related and cellular growth pathways Front Microbiol 6: 50, 2015.

32. Li YC, He SM, He ZX, Li M, Yang Y, Pang JX, Zhang X, Chow K, Zhou Q, Duan W, et al: Plumbagin induces apoptotic and autophagic cell death through inhibition of the PI3K/Akt/mTOR pathway in human non-small cell lung cancer cells. Cancer Lett 344: 239-259, 2014. 